Document downloaded from:

http://hdl.handle.net/10251/64663

This paper must be cited as:

Gregori Gregori, V.; Miñana, JJ. (2015). std-Convergence in fuzzy metric spaces. Fuzzy Sets and Systems. 267:140-143. doi:10.1016/j.fss.2014.05.007.

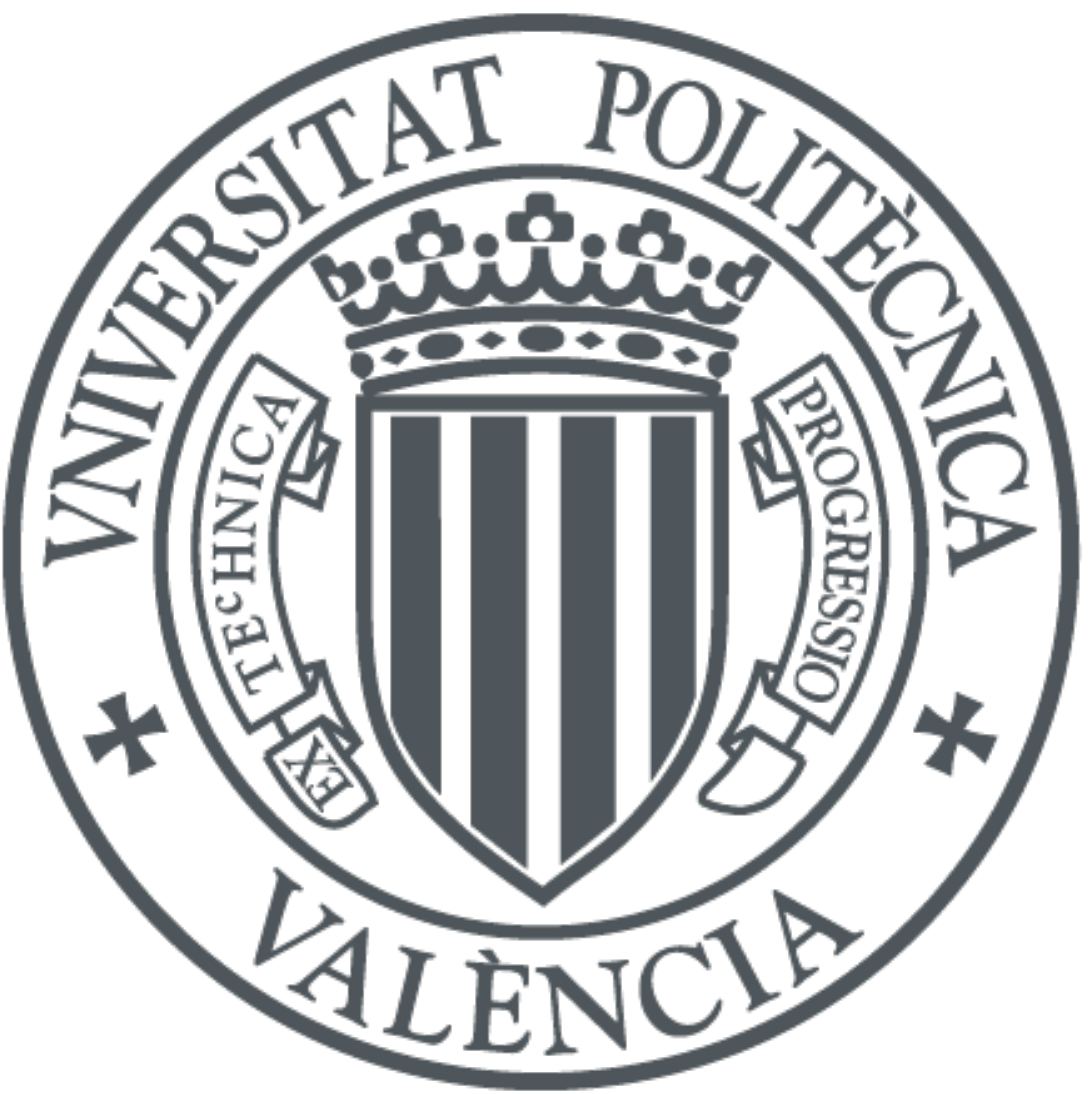

The final publication is available at

http://dx.doi.org/10.1016/j.fss.2014.05.007

Copyright Elsevier

Additional Information 
1

2

3

\title{
std-convergence in fuzzy metric spaces
}

\author{
Valentín Gregori ${ }^{\mathrm{a}, *, 1}$, Juan-José Miñana ${ }^{\mathrm{a}, \mathrm{b}, 2}$ \\ a Instituto Universitario de Matemática Pura y Aplicada, Universidad Politécnica \\ de Valencia, Camino de Vera $s / n$ 46022 Valencia (SPAIN). \\ bjuamiapr@upvnet.upv.es
}

\begin{abstract}
In this note we answer two recent questions posed by Morillas and Sapena [On Cauchy sequences in fuzzy metric spaces, Proceedings of the Conference in Applied Topology WiAT'13 101-108] related to standard convergence in fuzzy metric spaces in the sense of George and Veeramani. The obtained results lead us to establish what conditions must satisfy a concept about sequential convergence to be considered compatible with a concept of Cauchyness.
\end{abstract}

Key words: Fuzzy metric space; (std-) Cauchy sequence; (std-) convergent sequence.

\section{Introduction}

Kramosil and Michalek gave in [8] a concept of fuzzy metric space which is an extension of the concept of Menger space to the fuzzy setting. A more general version of this concept, denoted by $K M$-fuzzy metric space, was given later

* Corresponding author email: vgregori@mat.upv.es; telephone/fax number: +034 962849300

1 Valentín Gregori acknowledges the support of Spanish Ministry of Education and Science under Grant MTM 2012-37894-C02-01 and the supports of Universitat Politècnica de València under Grant PAID-05-12 SP20120696 and under Grant PAID-06-12 SP20120471.

2 Juan José Miñana acknowledges the support of Conselleria de Educación, Formación y Empleo (Programa Vali+d para investigadores en formación) of Generalitat Valenciana, Spain and the support of Universitat Politècnica de València under Grant PAID-06-12 SP20120471. 
$([2,3])$. In this note we deal with the concept of fuzzy metric space due to George and Veeramani (Definition 1) which is a modification of the concept of $K M$-fuzzy metric space.

A significant difference between fuzzy metric and metric is that the first one includes in its definition a $t$-parameter. From the mathematical point of view the $t$-parameter allows to introduce novel (fuzzy) metric concepts with respect to the classical metric ones (and sometimes the constructed fuzzy theory is more general than the corresponding one in classical theory). For instance, several well-motivated notions of convergence and Cauchyness related to sequences can be found in the literature $[2-4,9-11,13]$. In particular, more recently a stronger concept than convergence, called $s$-convergence, has been used to characterize certain type of fuzzy metric spaces [5]. Then, for a concept of convergence it is natural and interesting to study a concept of Cauchyness, or vice-versa, such that both are pairwise compatible. This is not an original idea. In fact, it was already suggested by D. Mihet in [9] where the author defined a weaker concept than convergence, called $p$-convergence. Then, in [4] the authors gave (an appropriate) concept of $p$-Cauchy sequence and also they initiated the study on the relationship of the concept of $p$-convergence with local bases. This study has been continued in [6].

For establishing relationships between the theory of complete fuzzy metric spaces and domain theory, Ricarte and Romaguera have introduced in [11] a stronger concept than Cauchy sequence, called standard Cauchy, briefly stdCauchy. They have proved that the well-known theorem due to Edalat and Heckmann [1] that characterizes complete metric spaces by means of continuous domains can be obtained from their results in fuzzy metrics ([11], Corollary 1). Furthermore, the theory constructed in that paper cannot be obtained from the metric case. Indeed, if $M$ is a non-complete stationary fuzzy metric then it is $s t d$-complete but the uniformity $\mathcal{U}_{M}$ induced by $M$, see [7], is not complete and so all metrics compatible with $\mathcal{U}_{M}$ are not complete and then classical theory cannot be applied on $M$.

Inspired in the classical case the authors have introduced in [10], in a natural way, the concept of standard convergence, briefly std-convergence, and they have asked the following questions.

$Q 1$ : Is every std-convergent sequence a std-Cauchy sequence?

Q2 : Let $\left\{x_{n}\right\}$ be a std-Cauchy and convergent sequence. Is $\left\{x_{n}\right\}$ std-convergent?

In this note we give negative response to $Q 1$ in Example 5 and then we conclude that the concept of std-convergence is not appropriate. Then, for avoiding the proliferation of non-appropriate concepts related to convergence or Cauchyness, we create a framework in which the study of the relationship between both concepts to be more useful. So, we establish in Definition 7 when 
a concept of convergence is compatible with a concept of Cauchyness, and vice-versa. Later, we give a concept of convergence which is compatible with std-Cauchy. Finally, we give a positive answer to $Q 2$.

\section{Preliminaries}

Definition 1 (George and Veeramani [2]). A fuzzy metric space is an ordered triple $(X, M, *)$ such that $X$ is a (non-empty) set, $*$ is a continuous $t$-norm and $M$ is a fuzzy set on $X \times X \times] 0, \infty[$ satisfying the following conditions, for all $x, y, z \in X, s, t>0$ :

(GV1) $M(x, y, t)>0$

(GV2) $M(x, y, t)=1$ if and only if $x=y$;

(GV3) $M(x, y, t)=M(y, x, t)$;

$\left(G V_{4}\right) M(x, y, t) * M(y, z, s) \leq M(x, z, t+s)$

$(G V 5) M(x, y,-):] 0, \infty[\rightarrow] 0,1]$ is continuous.

Every fuzzy metric $M$ on $X$ generates a topology $\tau_{M}$ on $X$ which has as a base the family of open sets of the form $\left\{B_{M}(x, \epsilon, t): x \in X, \epsilon \in\right] 0,1[, t>0\}$, where $B_{M}(x, \epsilon, t)=\{y \in X: M(x, y, t)>1-\epsilon\}$ for all $\left.x \in X, \epsilon \in\right] 0,1[, t>0$.

Let $(X, d)$ be a metric space and let $M_{d}$ a function on $\left.X \times X \times\right] 0, \infty[$ defined by

$$
M_{d}(x, y, t)=\frac{t}{t+d(x, y)}
$$

Then $\left(X, M_{d}, \cdot\right)$ is a fuzzy metric space, [2], and $M_{d}$ is called the standard fuzzy metric induced by $d$. The topology $\tau_{M_{d}}$ coincides with the topology $\tau(d)$ on $X$ deduced from $d$.

Definition 2 (George and Veeramani [2]). A sequence $\left\{x_{n}\right\}$ in a fuzzy metric space $(X, M, *)$ is called Cauchy if given $\epsilon \in] 0,1\left[\right.$ and $t>0$ there exists $n_{0} \in \mathbb{N}$ such that $M\left(x_{n}, x_{m}, t\right)>1-\epsilon$ for all $n, m \geq n_{0} . X$ is called complete if every Cauchy sequence in $X$ is convergent.

Definition 3 (Ricarte and Romaguera [11]). A sequence $\left\{x_{n}\right\}$ is called stdCauchy if given $\epsilon \in] 0,1\left[\right.$ there exists $n_{\epsilon} \in \mathbb{N}$, depending on $\epsilon$, such that $M\left(x_{n}, x_{m}, t\right)>\frac{t}{t+\epsilon}$, for all $n, m \geq n_{\epsilon}$ and for all $t>0 . X$ is called stdcomplete if every std-Cauchy sequence in $X$ is convergent.

Definition 4 (Morillas and Sapena [10]). A sequence $\left\{x_{n}\right\}$ in $X$ is called std-convergent to $x_{0} \in X$ if given $\left.\epsilon \in\right] 0,1\left[\right.$ there exists $n_{\epsilon} \in \mathbb{N}$, depending on $\epsilon$, such that $M\left(x_{n}, x_{0}, t\right)>\frac{t}{t+\epsilon}$, for all $n \geq n_{\epsilon}$ and for all $t>0$. 


\section{$3 \quad$ Results}

The next example gives a negative response to the first question Q1.

Example 5 (A std-convergent non-std-Cauchy sequence). Let $d$ be the usual metric on $\mathbb{R}$ restricted to $[0, \infty[$ and consider the standard fuzzy metric induced by $d$. Let $X=[0, \infty[$. We define on $X \times X \times] 0, \infty[$ the function

$$
M(x, y, t)= \begin{cases}1, & \text { if } x=y \\ M_{d}(x, 0, t) \cdot M_{d}(0, y, t), & \text { if } x \neq y\end{cases}
$$

It is an easy exercise to prove that $(X, M, \cdot)$ is a fuzzy metric space.

Now, consider the sequence $\left\{x_{n}\right\}$ in $X$, where $x_{n}=\frac{1}{n}$ for all $n \in \mathbb{N}$. We claim that $\left\{x_{n}\right\}$ is std-convergent to 0 . Indeed, take $\left.\epsilon \in\right] 0,1\left[\right.$, then we can find $n_{\epsilon} \in \mathbb{N}$ such that $n_{\epsilon}>\frac{1}{\epsilon}$ and hence $M\left(x_{n}, 0, t\right)=\frac{t}{t+\frac{1}{n}}>\frac{t}{t+\epsilon}$, for all $n \geq n_{\epsilon}$ and for all $t>0$. So $\left\{x_{n}\right\}$ is std-convergent to 0 .

We claim that $\left\{x_{n}\right\}$ is not std-Cauchy. Indeed, if we suppose that $\left\{x_{n}\right\}$ is std-Cauchy, then for each $\epsilon \in] 0,1\left[\right.$ there exists $n_{\epsilon} \in \mathbb{N}$ such that

$$
M\left(x_{n}, x_{m}, t\right)=\frac{t}{t+\frac{1}{n}} \cdot \frac{t}{t+\frac{1}{m}}>\frac{t}{t+\epsilon}
$$

for all $n, m \geq n_{\epsilon}$ and $t>0$. So, $\frac{t}{\left(t+\frac{1}{n_{\epsilon}}\right)\left(t+\frac{1}{n_{\epsilon}}\right)}>\frac{1}{t+\epsilon}$, for all $t>0$.

Then, $\lim _{t \rightarrow 0} \frac{t}{\left(t+\frac{1}{n_{\epsilon}}\right)\left(t+\frac{1}{n_{\epsilon}}\right)}=0 \geq \lim _{t \rightarrow 0} \frac{1}{t+\epsilon}=\frac{1}{\epsilon}$, a contradiction.

Remark 6 Attending to Definition 3 it is clear that a natural way of defining std-convergence is the one given by the authors in [10] (Definition 4). Unfortunately, as shows Example 5, this definition should be considered not appropriate.

Next we establish conditions under which a pair of concepts on convergence and Cauchyness, related to sequences, are considered pairwise compatible. These conditions have been chosen for preserving the natural structure among the concepts and also, for avoiding the unnecessary appearance of concepts or inner properties (which, finally, could distort the next diagrams).

Definition 7 Suppose it is given a sequential stronger (weaker, respectively) concept than Cauchy, say s-Cauchy (w-Cauchy, respectively). A concept on convergence, say s-convergence (w-convergence, respectively), is said to be compatible with s-Cauchy (w-Cauchy, respectively), and vice-versa, if the di- 
agram of implications below on the left (on the right, respectively) is fulfilled

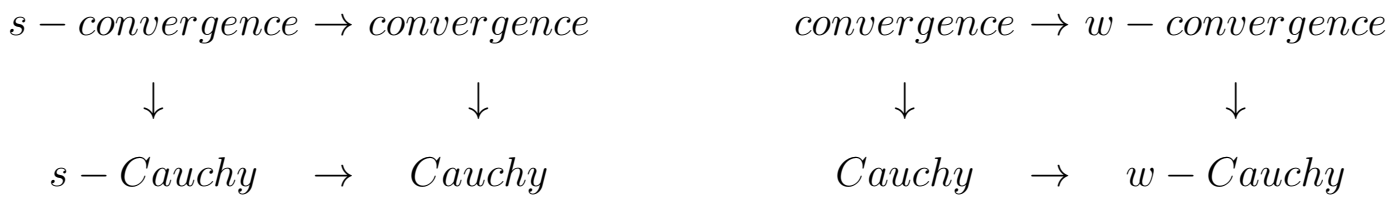

So, by Example 5 we can assert that the concept of std-convergence is not compatible with std-Cauchy. After the next remark we give a concept of convergence which is compatible with std-Cauchy.

Remark 8 (Existence of pairwise compatible s-concepts). Suppose that a concept of s-Cauchyness which is stronger than Cauchy, is given. Also, suppose that there is not any implication between convergence and s-Cauchyness. Then, there exists a concept of s-convergence compatible with s-Cauchy if and only if s-Cauchy and convergence are non-mutually exclusive concepts. Indeed, in a such case we can give the next definition: A sequence $\left\{x_{n}\right\}$ is called $s^{*}$-convergent if it is convergent and s-Cauchy. Obviously, this concept of $s^{*}$-convergence is compatible with s-Cauchy. Further, any concept of sconvergence which is compatible with $s$-Cauchy is stronger than $s^{*}$-convergence.

Now, since every std-convergent sequence is convergent, [10], then Example 5 provides an example of a convergent sequence which is not std-Cauchy. On the other hand if $\left(X, M_{d}, \cdot\right)$ is a standard fuzzy metric then a sequence in $X$ is std-Cauchy if and only if it is Cauchy. Hence, in a non-complete standard fuzzy metric space we can find std-Cauchy sequences which are not convergent. Further, every convergent sequence in $\left(X, M_{d}, \cdot\right)$ is std-Cauchy. Thus, by the last remark we can introduce the following definition of convergence which is compatible with std-Cauchy.

Definition 9 A sequence is called std ${ }^{*}$-convergent if it is convergent and stdCauchy.

Remark 10 (Existence of pairwise compatible w-concepts). Suppose that a concept of w-convergence which is weaker than convergence is given. Also, suppose that there is not any implication between w-convergence and Cauchy. Then, we always can find concepts of Cauchyness compatible with w-convergence. Indeed, in a such case we can give the next definition: $\left\{x_{n}\right\}$ is called $w^{*}$-Cauchy if $\left\{x_{n}\right\}$ is Cauchy or $w$-convergent. Clearly, $w^{*}$-Cauchy is compatible with $w$ convergence. Further, any other concept of w-Cauchy which is compatible with w-convergence is weaker than $w^{*}$-Cauchy.

Finally, in the next proposition we response in a positive way to Question $Q 2$. 
Proposition 11 Let $(X, M, *)$ be a fuzzy metric space and let $\left\{x_{n}\right\}$ be a stdCauchy convergent sequence. Then $\left\{x_{n}\right\}$ is std-convergent.

\section{Proof.}

Let $\left\{x_{n}\right\}$ be a std-Cauchy convergent sequence. Fix $\left.\epsilon \in\right] 0,1[$ and $t>0$. Suppose that $\left\{x_{n}\right\}$ converges to $x_{0}$. Since $M(x, y,-)$ is continuous for all $x, y \in X$, by Corollary 7.2 of [3] (or using Proposition 1 of [12]) we have that $\lim _{m} M\left(x_{n}, x_{m}, t\right)=M\left(x_{n}, x_{0}, t\right)$ for all $n \in \mathbb{N}$.

On the other hand, since $\left\{x_{n}\right\}$ is std-Cauchy we have that for $\left.\delta \in\right] 0, \epsilon$ there exists $n_{\delta} \in \mathbb{N}$ such that

$$
M\left(x_{n}, x_{m}, t\right)>\frac{t}{t+\delta}>\frac{t}{t+\epsilon}, \text { for all } n, m \geq n_{\delta} \text { and all } t>0 .
$$

Then

$$
M\left(x_{n}, x_{0}, t\right)=\lim _{m} M\left(x_{n}, x_{m}, t\right) \geq \frac{t}{t+\delta}>\frac{t}{t+\epsilon}, \text { for all } n \geq n_{\delta} \text { and all } t>0
$$

and so $\left\{x_{n}\right\}$ is std-convergent.

\section{Acknowledgements}

The authors are grateful to the referees for their valuable suggestions.

\section{References}

[1] A. Edalat, R. Heckmann, A computational model for metric spaces, Theoretical Computer Science 193 (1998) 53-73.

[2] A. George, P. Veeramani, On some results in fuzzy metric spaces, Fuzzy Sets and Systems 64 (1994) 395-399.

[3] M. Grabiec, Fixed points in fuzzy metric spaces, Fuzzy Sets and Systems 27 (1989) 385-389.

[4] V. Gregori, A. López-Crevillén, S. Morillas, A. Sapena, On convergence in fuzzy metric spaces, Topology and its Applications 156 (2009) 3002-3006.

[5] V. Gregori, J. J. Miñana, S. Morillas, A note on convergence in fuzzy metric spaces, submitted to Iranian Journal of Fuzzy Systems. 
[6] V. Gregori, J. J. Miñana, S. Morillas, A note on local bases and convergence in fuzzy metric spaces, Topology and its Applications 163 (2014) 142-148.

[7] V. Gregori, S. Romaguera, Some properties of fuzzy metric spaces, Fuzzy Sets and Systems 115 (2000) 485-489.

[8] I. Kramosil, J. Michalek, Fuzzy metrics and statistical metric spaces, Kybernetika 11 (1975) 326-334.

[9] D. Mihet, On fuzzy contractive mappings in fuzzy metric spaces, Fuzzy Sets and Systems 158 (2007) 915-921.

[10] S. Morillas, A. Sapena, On standard Cauchy sequences in fuzzy metric spaces, Proceedings of the Conference in Applied Topology WiAT'13 101-108.

[11] L. A. Ricarte, S. Romaguera, A domain-theoretic approach to fuzzy metric spaces, Topology and its Applications 163 (2014) 149-159.

[12] J. Rodríguez-López, S. Romaguera, The Hausdorff fuzzy metric on compact sets, Fuzzy Sets and Systems 147 (2004) 273-283.

[13] H. Sherwood, On the completion of probabilistic metric spaces, Z. Wahrscheinlichkeitstheorie verw. Geb. 6 (1966) 62-64. 